\title{
Sample Manipulation and Data Assembly for Robust Microcrystal Synchrotron Crystallography
}

Qun Liu,a,b, Gongrui Guo ${ }^{a, b}$, Martin R. Fuchs ${ }^{a}$, Wuxian Shi ${ }^{a}$, John Skinner ${ }^{a}$, Evanna Berman ${ }^{b}$, Craig M. Ogata ${ }^{c}$, Wayne A. Hendrickson ${ }^{d}$, Sean McSweeney ${ }^{a}$

a Photon Science Directorate, NSLS-II, Brookhaven National Laboratory, Upton, NY 11973, USA

${ }^{b}$ Biology Department, Brookhaven National Laboratory, Upton, NY 11973, USA

'GM/CA@APS, X-Ray Science Division, Advanced Photon Source, Argonne National Laboratory, Argonne, IL 60439, USA

d Department of Biochemistry and Molecular Biophysics, Columbia University, New York, NY 10032, USA.

Contact email: qunliu@bnl.gov

With the recent development of microcrystal handling, synchrotron microdiffraction beamline instrumentation and data analysis, microcrystal crystallography with crystal sizes of sub-10 microns is appealing at synchrotrons. However, challenges remain in sample manipulation and data assembly for robust microcrystal synchrotron crystallography. Here we describe the development of micro-sized polyimide wellmounts for manipulation of microcrystals of a few microns and the implementation of a robust data analysis method for assembly of rotational microdiffraction datasets from many microcrystals. By progressively processing single-crystal datasets, by using a reference dataset, and by using iterative crystal and frame rejection, our data assembly procedure is quite robust. By combining these strategies, our method provides an attractive route for optimized microcrystal crystallography experiments at synchrotrons. Beyond this, our data assembly strategy may work equally well for microdiffraction from larger crystals. 